\title{
O MERCOSUL E A INTEGRAÇÃO REGIONAL DOS MERCADOS ACIONÁRIOS ARGENTINO E BRASILEIRO
}

\author{
Ricardo Pereira Câmara Leal \\ Professor Adjunto de Finanças e Coordenador do Centro de Estudos \\ e Pesquisas em Seguros da COPPEAD/UFRJ, Doutor pela \\ COPPEAD/UFRJ e Editor da Latin American Business Review e Latin \\ American Economic Abstracts. \\ E-mail: rleal@coppead.ufrj.br
}

Giovani Pereira e Silva Operador de Câmbio do Banco da Bahia de Investimentos e Mestre em Administração pela PUC-RJ.

RESUMO: Este estudo analisa o comportamento recente dos mercados acionários argentino e brasileiro. Nosso objetivo é avaliar o nível de integração entre esses mercados nos períodos anterior (1982-1990) e posterior (19911995) à criação do Mercosul. Os resultados mostram clara distinção entre os dois períodos analisados. No período anterior ao Mercosul, os mercados latinos encontravam-se segmentados, não mantendo qualquer relação com os fatores globais que utilizamos. Para o período posterior ao Mercosul, encontramos evidência de que os mercados argentino e brasileiro estão mais integrados entre si, mesmo depois de se controlar para a integração com os mercados americano e chileno.

ABSTRACT: This study investigates the recent behavior of the Argentine and Brazilian stock markets. Our goal is to evaluate the level of integration between these markets in the period prior (1982-1990) and after (1991-1995) the introduction of Mercosul. Our findings show a clear distinction between the two periods. Before the introduction of Mercosul, the Latin markets were segmented and were not related to the global pricing factors we used. For the period after the introduction of Mercosul, we found evidence of increased integration between the Argentine and Brazilian stock markets even after we controlled for integration with the US and Chilean markets.

PALAVRAS-CHAVE: mercados emergentes, integração de mercados, Mercosul, Argentina, Brasil.

KEY WORDS: emerging markets, market integration, Mercosul, Argentina, Brazil. 
Os mercados de ações dos países em desenvolvimento têm experimentado expressivas mudanças nos últimos anos. Segundo Mullin, ${ }^{1}$ entre 1989 e 1992 os valores do investimento em carteira em mercados emergentes triplicaram. Muito da atratividade dos mercados de ações em países em desenvolvimento deriva dos elevados retornos que apresentaram nos últimos anos. ${ }^{2}$ A questão

\section{PROCURAREMOS EXAMINAR SE ESSES DOIS GRANDES MERCADOS EMERGENTES - ARGENTINA E BRASIL - ESTÃO MAIS PRÓXIMOS UM DO OUTRO DEVIDO AO MOVIMENTO MUNDIAL DE LIBERALIZAÇÃO DOS MERCADOS ACIONÁRIOS, APROXIMANDO-OS DOS MERCADOS PRINCIPAIS, EM PARTICULAR DOS EUA, OU SE SUA APROXIMAÇÃO SE DEVE A FATORES REGIONAIS.}

dos elos e da integração entre os mercados tem implicações importantes para os benefícios da diversificação internacional. À medida que os mercados se tornam mais próximos economicamente, mais eles tenderão a mover-se em conjunto. Um fato recente como o discurso do diretor do Federal Reserve, Alan Greenspan, na noite de 5 de dezembro de 1996, interpretado como uma possível indicação da elevação das taxas de juros americanas, levou os mercados asiáticos e europeus a caírem, desaguando em forte queda do mercado americano, que abre depois daqueles devido aos fusos horários. A crise cambial mexicana e o chamado "Efeito Tequila" constituem outro exemplo desse contágio entre os mercados. Quando os mercados se tornam mais integrados, uma crise num mercado pode se propagar para outros, diminuindo os benefícios do investimento internacional.

Nosso propósito é o de estudar se os mercados acionários da Argentina e do Brasil parecem estar mais integrados. A recente aproximação dos dois mercados é conhecida, resultando na união aduaneira do Mercosul. Por outro lado, esses mercados se abriram ao investidor estrangeiro independentemente um do outro. Procuraremos examinar se esses dois grandes mercados emergentes estão mais próximos um do outro devido ao movimento mundial de liberalização dos mercados acionários, aproximando-os dos mercados principais, em particular dos EUA, ou se sua aproximação se deve a fatores regionais. Utilizamos o mercado chileno para o controle para fatores regionais. $\mathrm{O}$ Chile, apesar de não ser membro do Mercosul, ${ }^{3}$ tem investido nas economias da Argentina e do Brasil. Se, depois do controle para fatores globais e regionais, ainda verificarmos uma relação entre os mercados argentino e brasileiro, isso será evidência de que esses mercados estão se integrando entre si, e um dos fatores primordiais para essa integração é sem dúvida o Mercosul. Nossos resultados, descritos em detalhe a seguir, sustentam essa afirmativa.

\section{FATORES QUE CONTRIBUEM PARA A INTEGRAÇÃO DOS MERCADOS}

As reformas básicas da economia, apoiadas em programas que contemplam a estabilização das taxas de juros e o controle inflacionário, são pontos de grande relevância para o investimento estrangeiro. Programas de privatização ambiciosos têm aumentado o influxo de capital, melhorando o balanço fiscal e promovendo a estabilidade macroeconômica. O governo argentino iniciou um programa de estabilização a partir de 1990, balanceando suas contas fiscais, e, em abril de 1991, atrelou o peso ao dólar americano para reduzir a inflação doméstica. O sucesso na renegociação da reestruturação da dívida com os bancos comerciais, na linha do Plano Brady, aumentou a confiança dos investidores no país. O Brasil também apresentou grandes entradas de capitais, apesar das altas taxas de inflação e da instabilidade econômica. Em 1992, o País reestruturou sua dívida junto ao Clube de Paris, assinando um acordo stand-by com o Fundo Monetário Internacional e, em abril de 1994, promoveu a revisão da dívida junto aos banqueiros internacionais, também de acordo com o Plano Brady. Em julho de 1994, houve a instauração do Plano Real e, em 1995, o País já trabalhava com níveis in- 
flacionários aceitáveis pela comunidade financeira internacional. $\mathrm{O}$ crescente influxo de recursos externos atesta a crescente confiança do investidor internacional no mer- econômico internacional. ${ }^{11}$ Nosso trabalho procura analisar o impacto do acordo Mercosul no nível de integração dos mercados acionários da Argentina e do Brasil.

Tabela 1 - Fluxos de capitais para a América Latina (em bilhões de dólares)

\begin{tabular}{|c|c|c|c|c|c|}
\hline Ano & Direto & $\%$ & Carteira & $\%$ & Total \\
\hline 1987 & 4,7 & 57,1 & 3,5 & 42,9 & 8,3 \\
\hline 1988 & 6,9 & 95,9 & 0,3 & 4,1 & 7,2 \\
\hline 1989 & 6,7 & nd & $-7,7$ & nd & $-1,0$ \\
\hline 1990 & 6,0 & 63,6 & 3,4 & 36,4 & 9,4 \\
\hline 1991 & 8,7 & 45,7 & 10,3 & 54,3 & 19,0 \\
\hline 1992 & 10,9 & 43,5 & 14,1 & 56,5 & 25,0 \\
\hline 1993 & 13,2 & 24,3 & 41,1 & 75,7 & 54,3 \\
\hline 1994 & 15,4 & 43,3 & 20,2 & 56,7 & 35,7 \\
\hline $\begin{array}{l}\text { Fonte: Merrill Lynch, Quarterly Economic Outlook, 1996. } \\
\text { Nota: "nd" significa não-disponível. }\end{array}$ \\
\hline
\end{tabular}

cado brasileiro. A Tabela 1 apresenta o fluxo de capitais para a América Latina no período compreendido entre 1987 e 1994.

A abertura dos mercados também tem sido de fundamental importância para o aumento do interesse externo. ${ }^{4}$ Hargis ${ }^{5}$ apresenta evidência dos benefícios das medidas liberalizantes com foco na emissão de ADRs,${ }^{6}$ indicando melhoria na liquidez dos mercados e redução da volatilidade. Beckaert \& Harvey ${ }^{7}$ indicam que as medidas liberalizantes aumentam a correlação entre os mercados, mas não parecem alterar a volatilidade dos mercados emergentes. Aggarwal, Inclan \& $\mathrm{Leal}^{8}$ sugerem que as mudanças de volatilidade nos mercados emergentes devem-se primordialmente a choques locais e não parecem relacionadas com a abertura dos mercados.

As relações entre os diversos países do mundo vão, aos poucos, cedendo lugar às negociações multilaterais. A existência de um bloco econômico sugere que os mercados nos respectivos países membros apresentam maior correlação. Assim, seria razoável esperar que os mercados membros da União Européia tendam a apresentar maior correlação entre si. O mesmo poderia ser dito para os EUA, México e Canadá, em função do NAFTA. ${ }^{9}$ Em termos de América do Sul, a criação do Mercosul faz parte de um processo evolutivo dos países do cone sul ${ }^{10}$ que vêm procurando criar condições necessárias à inserção de seus membros no cenário

\section{MERCADOS EMERGENTES E A QUESTÃO DA INTEGRAÇÃO}

Harvey ${ }^{12}$ avalia que os retornos das ações em mercados emergentes mantêm relação positiva com a performance econômica, em que se destacam indicadores como a taxa de crescimento das exportações, a taxa de pagamento de dividendos, medidas de atividade econômica, entre outras. Segundo Harvey, ${ }^{13}$ muitos mercados emergentes ainda mantêm um significativo grau de segmentação em relação a diversos fatores globais e são explicados primordialmente por fatores locais. Por outro lado, Harvey e Beckaert \& Harvey ${ }^{14}$ constatam a importância crescente de fatores globais para explicar o comportamento dos retornos e da volatilidade em vários mercados emergentes. Mullin ${ }^{15}$ sugere que os retornos das ações de mercados emergentes são correlacionados com variáveis macroeconômicas locais e com a abertura da economia representada pela taxa de crescimento de exportações.

Solnik et aliii ${ }^{16}$ examinam a relação entre as correlações entre os grandes mercados nacionais de ações e a volatilidade. Um fato constatado por Solnik et alii é que há uma relação entre a volatilidade e o nível de correlações. O comportamento dos mercados é em grande parte explicado por fatores locais, mas eles tendem a se mover em conjunto com outros mercados nacionais quando choques negativos importantes ocorrem. Por exem-
4. Para um sumário das medidas liberalizantes nos diversos mercados emergentes, veja Beckaert \& Harvey (no prelo) e, para os mercados latinos, veja Hargis (1996).

5. HARGIS, Kent. ADRs in emerging markets: market competition or fragmentation. University of South Carolina (Working Paper), 1996

6. 0 ADR ou American Depositary Receipt é um certificado sobre ações de uma empresa não-americana custodiadas por um trust que negocia nos EUA sem ter de passar pelo processo completo de disclosure exigido pela Comissão de Valores Mobiliários americana. A negociação do ADR pode ser restrita a certos investidores institucionais, e a emissão de novos títulos no mercado doméstico americano pode não ser permitida, dependendo do tipo de ADR.

7. BECKAERT, Geert, HARVEY, Campbell. Emerging equity market volatility. Journal of Financial Economics, no prelo.

8. AGGARWAL, Reena, INCLAN, Carla, LEAL, Ricardo. Volatility in emerging stock markets. Georgetown University (Working Paper), 1996.

9. NAFTA, North American Free Trade Agreement ou Acordo de Livre Comércio da América do Norte.

10. 0 cone sul foi assim denominado devido à localização geográfica de seus membros, formando um cone invertido, incluindo, além dos países signatários do Mercosul, o Chile e a Bolívia.

11. Para uma análise da integração hemisférica, veja VELLOSO, João Paulo dos R. Mercosul e o Nafta: O Brasil e a Integração Hemisférica. Rio de Janeiro: José Olympio, 1995.

12. HARVEY Campbell. Predictable risk and returns in emerging markets. Review of Financial Studies, v.8, p.773-816, 1995.

13. Idem, ibidem.

14. Idem, ibidem; BECKAERT, Geert, HARVEY, Campbell. Op. cit.

15. MULLIN, J. Op. cit.

16. SOLNIK, Bruno, BOUCRELLE, Cyril, LE FUR, Yann. International market correlation and volatility. Financial Analysts Journal, Sep.-Oct., p.17-34, 1996. 
plo, Roll ${ }^{17}$ reporta que, anteriormente ao crash de 1987, as correlações entre mercados eram relativamente baixas. Outubro de 1987 foi o único mês, durante a década de 80 , em que grande parte dos mercados se

\section{OS DADOS DIÁRIOS DOS ÍNDICES \\ UTILIZADOS ENVOLVEM O PERÍODO ENTRE JANEIRO DE 1986 E DEZEMBRO DE 1995. ESSE PERÍODO FOI ESCOLHIDO POR CONSIDERARMOS O INÍCIO DO ANO DE 1991 COMO MARCO RELEVANTE PARA A ANÁLISE DAS MUDANÇAS NO CONE SUL. A ESCOLHA DO ANO DE 1991 DEVE-SE À ASSINATURA DO TRATADO DE ASSUNÇÃO EM MARÇO DE 1991.}

17. ROLL, Richard. Price volatillity, international markets links, and their implications for regulatory policies. Journal of Financial Services Research, v.3, p.113-47, 1989

18. RATNER, Mitchell, LEAL, Ricardo. Casuality tests for the emerging markets of Latin America. Journal of Emerging Markets, v.1, n.1, p.29-40, 1996.

19. Idem, ibidem; SOLNIK et al. Op. cit.; DIVECHA, B. et al. Emerging markets: a quantitative perspective. Journal of Portfolio Management, 19, p.41-56, 1992; ZANETTE, Jorge J. Os mercados emergentes na formação de portfólios internacionais: um estudo empírico do Brasil e da Argentina. Revista Brasileira de Administração Contemporânea, v.19, p.341-61, 1995.

20. HARVEY, C. Op. cit.

21. BECKAERT, G., HARVEY, C. Op. cit.

22. HARVEY, C. Op. cit.; RATNER, M., LEAL, R. Op. cit.

23. HARVEY, C. Op. cit.

24. IFC, Emerging Stock Markets Factbook. IFC: Washington, DC, 1996. Merrill Lynch Economic Research, Quarterly Economic Outlook, March 1996. moveu na mesma direção. Em geral, em períodos de crise mundial, as correlações tendem a aumentar. Isso pôde ser observado também durante o embargo da OPEC (países exportadores de petróleo) no período entre julho de 1973 e abril de 1974. Segundo Ratner \& Leal, ${ }^{18}$ a relação contemporânea entre os mercados latinos e o mercado americano parece estar se tornando mais intensa.

É importante conceituar o que é a integração entre mercados de capitais. A integração de mercados na ótica financeira significa que o preço de ativos na mesma classe de risco será o mesmo em diferentes mercados. Cabe ressaltar a diferença entre o conceito de integração de mercados e o conceito de elos entre mercados. Os elos refletem o relacionamento entre mercados e podem ser evidenciados por coeficientes de correlação. Dois mercados podem ser correlacionados sem serem necessariamente integrados uma vez que seu movimento conjunto pode ser causado por um fator comum externo a ambos. A integração é um conceito mais amplo e deve ser investigada a partir de modelos de precificação de ativos. Os diversos estudos de correlação entre os mercados ${ }^{19}$ estudam os elos entre eles. Estudos como os de Harvey ${ }^{20}$ e Beckaert \& Harvey $^{21}$ desenvolvem modelos empíricos de apreçamento de ativos incluindo diversos fatores locais e globais e representam estudos sobre a integração entre os mercados. Finalmente, é muito possível que uma maior integração entre dois mercados se traduza em uma maior correlação entre eles, entretanto, o inverso não é necessariamente verdadeiro.

De forma geral, tanto os estudos sobre os elos de mercado quanto os estudos sobre a integração entre os mercados concluem em favor de um nível crescente de relacionamento e de integração entre os mercados emergentes e os grandes mercados internacionais, como o americano. ${ }^{22}$ Entretanto, a capacidade explicativa dos fatores locais ainda é predominante entre os mercados emergentes, indicando um significativo nível de segmentação nesses mercados com importantes benefícios para o investidor internacional. ${ }^{23}$

\section{METODOLOGIA}

A amostra analisada no presente estudo foi constituída pelos dois maiores mercados do Mercosul: Brasil e Argentina. Foram usados também os índices do mercado americano, mundial e chileno. Ao final de 1995, o mercado brasileiro, o maior mercado do Mercosul, era 46 vezes menor do que o mercado americano. O mercado argentino era 181 vezes menor que o americano e 4 vezes menor que o brasileiro. No final de 1995, o mercado brasileiro era o $19^{\circ}$ do mundo em termos de capitalização de mercado, enquanto o mercado argentino era o $32^{\circ} .{ }^{24} \mathrm{~A}$ capitalização, ao final de 1995, era de US\$ 148 bilhões para o Brasil e de US\$ 38 bilhões para a Argentina. A capitalização do mercado chileno, no fim de 1995, era de US\$ 74 bilhões, quase que o dobro da capitalização do mercado argentino e com cerca da metade do tamanho da capitalização do mercado brasileiro.

Os dados diários dos índices utilizados envolvem o período entre janeiro de 1986 e dezembro de 1995. Esse período foi escolhido por considerarmos o início do ano de 1991 como marco relevante para análise das mudanças no cone sul. A escolha do ano de 1991 deve-se à assinatura do Tratado de Assunção em março de 1991. Consideramos que a inclusão dos meses de ja- 
neiro e fevereiro de 1991 não representa um problema uma vez que os agentes econômicos já dispunham da informação da assinatura do tratado mesmo antes do início de 1991. A partir desse critério, estabeleceu-se a série histórica retrocedendose cinco anos até 1986 e avançando-se mais cinco anos até 1995, com dois períodos de cinco anos antes e depois de janeiro de 1991. O período pré-Mercosul vai de janeiro de 1986 a dezembro de 1990 e o período pós-Mercosul vai de janeiro de 1991 até dezembro de 1995.

Os dados coletados foram os níveis de fechamento do Índice General (Buenos Aires), do índice Ibovespa (São Paulo), do índice IGPA (Santiago), do S\&P500, representando o mercado americano, e do Morgan Stanley World Index (MSWI), calculado pela Morgan Stanley International Capital, representando o mercado mundial. O índice mundial inclui 22 países. ${ }^{25}$ Utilizamos o MSWI em dólares americanos. Todos os índices, com exceção do Ibovespa, são ponderados pelo valor de mercado. O Ibovespa é ponderado pelo volume das ações constantes na carteira teórica, que varia periodicamente. As séries de retornos não incluíram dividendos e refletem somente ganhos de capital. Como o que se investigou foram efeitos nos níveis das séries e não efeitos de riqueza, acreditamos não ser essa uma limitação considerável.

Foram calculados retornos semanais em moeda local e em dólar. Os retornos em moeda local foram obtidos de acordo com a equação 1 e os retornos em dólar pela equação 2. Os retornos semanais foram calculados a partir das quartas-feiras ${ }^{26}$ e se referem a um número variável de dias para cada mercado. As taxas de câmbio para o dólar foram obtidas no Wall Street Journal. Quando não havia cotações para um determinado dia, devido a um feriado americano, a cotação do dia foi estimada pela média dos dois dias adjacentes.

$$
\begin{aligned}
& R L_{i, t}=100 \times\left[\frac{I_{i, t}}{I_{i, t-1}}-1\right] \\
& R D_{i, t}=100 \times\left[\left(\frac{I_{i, t}}{I_{i, t-1}} \div \frac{X_{i, t}}{X_{i, t-1}}\right)-1\right]
\end{aligned}
$$

Onde $R L_{i, t}$ e $R D_{i, t}$ são os retornos em moeda local e em dólares, respectivamente. $I_{i, t}$ é o nível de fechamento do índice na quarta-feira $t$ no país $i$. $X_{i, t}$ é a taxa de câmbio de uma determinada quarta-feira $t$ do país $i$, obtida a partir do Wall Street Journal. A variação cambial semanal foi calculada segundo a equação 3 .

$$
R X_{i, t}=100 \times\left[\frac{X_{i, t}}{X_{i, t-1}}-1\right]
$$

Onde $R X_{i, t}$ é a variação cambial na quarta-feira $t$ para o país $i$.

\section{ENQUANTO NO PERÍODO PRÉ-MERCOSUL APENAS O CHILE APRESENTAVA COEFICIENTES DE CORRELAÇÃO SIGNIFICATIVOS COM OS MERCADOS DESENVOLVIDOS, AINDA QUE BAIXOS, NO PERÍODO PÓS-MERCOSUL TOdOS OS MERCADOS APRESENTAM CORRELAÇÃO SIGNIFICATIVA COM OS MERCADOS DESENVOLVIDOS. A ARGENTINA E O BRASIL APRESENTAM CORRELAÇÕES MAIS ELEVADAS DO QUE O PRÓPRIO CHILE APÓS 1990.}

Para o estudo da integração dos mercados, construímos um modelo de precificação multi-índice simples com base nos resultados apresentados por Harvey ${ }^{27}$ Harvey construiu diversos modelos de precificação baseados em fatores locais e globais. Dos diversos fatores globais utilizados, dois se destacaram com maior poder explicativo: o índice de ações americano e a taxa de câmbio para o dólar. Como o nosso interesse é o de investigar se houve um aumento da integração entre os mercados de ações argentino e brasileiro, elaboramos o seguinte modelo:
25. Os países são: Austrália, Áustria, Bélgica, Canadá, Dinamarca, Finlândia, França, Alemanha, Holanda, Hong Kong, Irlanda, Itália, Japão, Malásia, Nova Zelândia, Noruega, Cingapura, Espanha, Suécia, Suíça, Reino Unido e EUA.

26. A escolha das quartas-feiras tem 0 intuito de atenuar o efeito fim de semana. Para maiores detalhes sobre anomalias, veja AGGARWAL, Reena, LEAL, Ricardo. Integration and anomalies in the emerging markets of Asia and Latin America. In: DOUKAS, J. e LANG, L. (Eds.). Research in International Business and Finance, Supplement 1, Greenwich, Conn.: JAI Press, p.75-99, 1996.

27. HARVEY, C. Op. cit. 


$$
\begin{aligned}
& R L_{A R G, t}=\alpha_{0}+\alpha_{1} R L_{A R G, \mathrm{t}-1}+\alpha_{2} R D_{E U A}+\alpha_{3} R L_{B R A}+\alpha_{4} X_{A R G}+\alpha_{5} R L_{C H I}+\varepsilon_{A R G, \mathrm{t}} \\
& R L_{B R A, t}=\alpha_{0}+\alpha_{1} R L_{B R A, \mathrm{t}-1}+\alpha_{2} R D_{E U A}+\alpha_{3} R L_{A R G}+\alpha_{4} X_{B R A}+\alpha_{5} R L_{C H I}+\varepsilon_{B R A, \mathrm{t}}
\end{aligned}
$$

$\alpha_{n}$ é um vetor de parâmetros, $R D_{E U A}$ é o S\&P500, $R L_{A R G}, R L_{B R A}$ e $R L_{C H I}$ são os retornos em moeda local dos índices General, Ibovespa e IGPA. $X_{i}$ é a mudança da taxa de câmbio de moeda local para dólares para a Argentina ou Brasil, conforme o caso. A variação cambial entra como um fator local representando a volatilidade da economia e a inflação. A inclusão do primeiro termo de auto-regressão deve-se à evidência de que esse termo tende a ser significativo em mercados emergentes. ${ }^{28}$

\section{APÓS A ASSINATURA DO TRATADO DE ASSUNÇÃO, MESMO CONTROLANDO PARA AS INTEGRAÇÕES GLOBAL, REPRESENTADA PELO MERCADO AMERICANO, E REGIONAL, REPRESENTADA PELO MERCADO CHILENO, O COEFICIENTE PARA O PAÍS PARCEIRO NO MERCOSUL É SIGNIFICATIVO TANTO PARA A ARGENTINA QUANTO PARA O BRASIL. ACREDITAMOS QUE ESSE RESULTADO SEJA EVIDÊNCIA DE QUE, ALÉM DE UMA MAIOR INTEGRAÇÃO GLOBAL E REGIONAL, OS MERCADOS ARGENTINO E BRASILEIRO ESTEJAM MAIS INTEGRADOS ENTRE SI.}

28. HARVEY, C. Op. cit.

29. Os testes $t$ que realizamos estão disponiveis com os autores. Testes de diferenças de média devem ser interpretados com cautela, uma vez que assumem propriedades distribucionais que podem não estar presentes na nossa amostra.

30. Esse teste está disponivel com os autores.

31. BECKAERT, G., HARVEY, C. Op. cit.; HARGIS, K. Op. cit.
Incluímos o mercado chileno para controlar efeitos de integração na região do cone sul esperando isolar os efeitos de integração entre Argentina e Brasil. O mercado americano foi incluído pela mesma razão, visando a isolar os efeitos de integração entre os mercados argentino e brasileiro com o mercado americano. Finalmente, numa segunda bateria de testes, substituímos o S\&P500 pelo MSWI para controlar para a possível integração com outros grandes mercados mundiais presentes no MSWI.
A análise estatística a seguir apresenta uma breve análise descritiva da amostra, uma análise de correlação, para abordar a questão dos elos entre mercados, e os resultados para o modelo de precificação definido acima para tratar da questão da integração entre mercados.

\section{APRESENTAÇÃO E DISCUSSÃO DOS RESULTADOS}

A Tabela 2 apresenta medidas descritivas para os períodos pré e pós-Mercosul e para o período total da amostra. Os retornos e a volatilidade para todos os países latinos são maiores no período pré-Mercosul. Isso não é surpreendente uma vez que as medidas bem-sucedidas de estabilização econômica para redução da inflação (Planos Cavallo e Real) foram introduzidas após janeiro de 1991. Estatísticas de diferenças de média ${ }^{29}$ não apresentadas nesse trabalho indicam que apenas os retornos médios e a variação cambial semanal média da Argentina são diferentes nos dois períodos. Isso se deve à coincidência da implementação do Plano Cavallo no período posterior à assinatura do Tratado de Assunção. Por outro lado, o teste de Levine $^{30}$ de diferença da variância entre os dois períodos sugere que a variância de todas as variáveis diminuiu significativamente no período pós-Mercosul exceto para a variação cambial semanal no Brasil. Enquanto o comportamento da volatilidade cambial pode ser explicado pelo período de introdução de planos bem-sucedidos de estabilização econômica (o Plano Real foi introduzido em 1994), a redução da volatilidade do índice de mercado em todos os mercados certamente está relacionada a um conjunto de fatores. Podemos enumerar desde o crash de 1987, que elevou a volatilidade dos S\&P500 e do MSWI no período pré-Mercosul, até a abertura dos mercados latinos, à qual muitos autores atribuem como causa de uma queda da volatilidade de mercado diante da menor segmentação. ${ }^{31}$

A Tabela 3 mostra o contraste entre os coeficientes de correlação nos períodos anterior e posterior ao Tratado de Assunção. 
As diferenças são marcantes. Enquanto no período pré-Mercosul apenas o Chile apresentava coeficientes de correlação significativos com os mercados desenvolvidos, ainda que baixos, no período pós-Mercosul todos os mercados apresentam correlação significativa com os mercados desenvolvidos. A Argentina e o Brasil apresentam correlações mais elevadas do que o próprio Chile após 1990. Não foi necessário eliminar o mês de outubro de 1987 da amostra préMercosul já que os coeficientes de correlação são insignificantes. A Tabela 3 ilustra a natureza dos elos entre os mercados argentino e brasileiro tanto entre si quanto com os demais mercados estudados. A Figura 1 ilustra a evolução anual dos coeficientes de correlação entre Argentina, Brasil e EUA. Por outro lado, o fato de as correlações terem aumentado pode tão somente significar que os mercados latinos estão mais integrados com o mercado americano sem necessariamente estarem mais integrados entre si. Dessa forma, passamos aos resultados dos modelos de apreçamento de ativos apresentados anteriormente.

A Tabela 4 apresenta os resultados para os modelos ARIMA $(1,0,0)$ para os retornos em moeda local para a Argentina e o Brasil nos três períodos estudados. Percebe-se que os coeficientes dos fatores globais e regionais (o mercado parceiro no Mercosul, o mercado americano ${ }^{32}$ e o mercado chileno) tornam-se significativos apenas no período pós-Mercosul. Após a assinatura do Tratado de Assunção, mesmo controlando para as integrações global, representada pelo mercado americano, e regional, representada pelo mercado chileno, o coeficiente para o país parceiro no Mercosul é significativo tan-

Tabela 2 - Estatísticas descritivas dos retornos semanais para períodos selecionados

\section{\begin{tabular}{|c|c|c|c|c|c|}
\hline Variável & Média & Desvio-padrão & Máximo & Mínimo & № de Observações \\
\hline
\end{tabular}}

Painel A: Período pré-Mercosul (janeiro de 1986 a dezembro de 1990)

\begin{tabular}{|l|r|r|r|r|r|}
\hline Argentina & 4,2 & 11,7 & 80,6 & $-33,6$ & 261 \\
\hline Brasil & 3,9 & 11,8 & 42,1 & $-46,0$ & 261 \\
\hline Chile & 1,0 & 3,3 & 11,0 & $-9,2$ & 261 \\
\hline EUA & 0,2 & 2,5 & 6,7 & $-15,4$ & 261 \\
\hline MSWI & 0,3 & 2,2 & 4,7 & $-13,5$ & 261 \\
\hline Câmbio Arg. & 4,0 & 12,5 & 140,0 & $-38,9$ & 261 \\
\hline Câmbio Br. & 3,9 & 4,8 & 31,1 & $-7,0$ & 261 \\
\hline
\end{tabular}

Painel B: Período pós-Mercosul (janeiro de 1991 a dezembro de 1995)

\begin{tabular}{|l|r|r|r|r|r|}
\hline Argentina & 1,0 & 6,9 & 54,9 & $-13,6$ & 261 \\
\hline Brasil & 5,2 & 9,5 & 36,2 & $-27,8$ & 261 \\
\hline Chile & 0,7 & 2,8 & 11,4 & $-10,6$ & 261 \\
\hline EUA & 0,2 & 1,5 & 5,5 & $-4,9$ & 261 \\
\hline MSWI & 0,2 & 1,5 & 5,8 & $-4,3$ & 261 \\
\hline Câmbio Arg. & 0,3 & 3,8 & 59,0 & $-4,8$ & 261 \\
\hline Câmbio Br. & 3,9 & 4,1 & 26,4 & $-9,0$ & 261 \\
\hline
\end{tabular}

Painel C: Período total (janeiro de 1986 a dezembro de 1995)

\begin{tabular}{|l|r|r|r|r|c|}
\hline Argentina & 2,6 & 9,7 & 80,6 & $-33,6$ & 522 \\
\hline Brasil & 4,5 & 10,7 & 42,1 & $-46,0$ & 522 \\
\hline Chile & 0,8 & 3,1 & 11,4 & $-10,6$ & 522 \\
\hline EUA & 0,2 & 2,0 & 6,7 & $-15,4$ & 522 \\
\hline MSWI & 0,2 & 1,9 & 5,8 & $-13,5$ & 522 \\
\hline Câmbio Arg. & 2,1 & 9,4 & 140,0 & $-38,9$ & 522 \\
\hline Câmbio Br. & 3,9 & 4,5 & 31,1 & $-9,0$ & 522 \\
\hline
\end{tabular}

Nota: Os retornos dos índices de mercado para Argentina, Brasil, Chile e EUA estão em moeda local. Os retornos do MSWI estão em dólares americanos. A variação semanal do câmbio foi calculada segundo a equação 3 . Todas as estatísticas estão em percentual.
32. Não reportamos aqui os resultados dos modelos que utilizam o MSWI, uma vez que levam às mesmas conclusões que os resultados com os modelos com 0 S\&P500. Os resultados com o MSWI estão disponíveis com os autores. 
to para a Argentina quanto para o Brasil. Acreditamos que esse resultado seja evidência de que, além de uma maior integração global e regional, os mercados argentino e brasileiro estão mais integrados entre si. Pelos coeficientes para o período a partir de
1991 pode-se notar que o mercado brasileiro é menos influente para o mercado argentino do que o mercado chileno e o americano. No caso brasileiro, o mercado mais influente é o americano, seguido do chileno e do argentino.

Tabela 3 - Correlação entre os índices de mercado selecionados para os períodos anterior e posterior ao Mercosul e para o período total

\begin{tabular}{|l|c|c|c|c|c|c|}
\hline & Argentina & Brasil & Chile & EUA & MSWI \\
\hline \multicolumn{2}{|l|}{ Painel A: Período pré-Mercosul (janeiro de 1986 a dezembro de 1990) } \\
\hline Argentina & 1,00 & 0,01 & 0,00 & 0,01 & $-0,08$ \\
\hline Brasil & 0,85 & 1,00 & 0,00 & 0,05 & 0,01 \\
\hline Chile & 0,99 & 0,94 & 1,00 & $\mathbf{0 , 2 2}$ & $\mathbf{0 , 1 6}$ \\
\hline EUA & 0,91 & 0,41 & 0,00 & 1,00 & $\mathbf{0 , 7 6}$ \\
\hline MSWI & 0,22 & 0,83 & 0,01 & 0,00 & 1,00 \\
\hline
\end{tabular}

Painel B: Período pós-Mercosul (janeiro de 1991 a dezembro de 1995)

\begin{tabular}{|l|l|l|l|l|l|}
\hline Argentina & 1,00 & $\mathbf{0 , 2 7}$ & $\mathbf{0 , 3 7}$ & $\mathbf{0 , 2 3}$ & $\mathbf{0 , 1 9}$ \\
\hline Brasil & 0,00 & 1,00 & $\mathbf{0 , 2 8}$ & $\mathbf{0 , 2 0}$ & $\mathbf{0 , 2 0}$ \\
\hline Chile & 0,00 & 0,00 & 1,00 & $\mathbf{0 , 1 4}$ & $\mathbf{0 , 1 6}$ \\
\hline EUA & 0,00 & 0,00 & 0,02 & 1,00 & $\mathbf{0 , 7 2}$ \\
\hline MSWI & 0,00 & 0,00 & 0,01 & 0,00 & 1,00 \\
\hline
\end{tabular}

Painel C: Período total (janeiro de 1986 a dezembro de 1995)

\begin{tabular}{|l|l|l|l|l|l|}
\hline Argentina & 1,00 & 0,08 & $\mathbf{0 , 1 3}$ & 0,06 & 0,00 \\
\hline Brasil & 0,06 & 1,00 & $\mathbf{0 , 1 1}$ & $\mathbf{0 , 1 0}$ & 0,08 \\
\hline Chile & 0,00 & 0,01 & 1,00 & $\mathbf{0 , 1 9}$ & $\mathbf{0 , 1 6}$ \\
\hline EUA & 0,14 & 0,02 & 0,00 & 1,00 & $\mathbf{0 , 7 4}$ \\
\hline MSWI & 0,93 & 0,07 & 0,00 & 0,00 & 1,00 \\
\hline
\end{tabular}

Nota: Os números acima da diagonal são os coeficientes de correlação. Os números abaixo da diagonal são os coeficientes de significância das correlações. Os coeficientes de correlação em negrito são significativos a um nível de $5 \%$.

Tabela 4 - Resultado para os modelos de integração com fatores locais e fatores globais

\begin{tabular}{|c|c|c|c|c|c|c|}
\hline Mercado & Constante & Primeira Defasagem & Parceiro & Chile & EUA & Câmbio \\
\hline \multicolumn{7}{|c|}{ Painel A: Período pré-Mercosul (janeiro de 1986 a dezembro de 1990) } \\
\hline Argentina & $0,028^{*}$ & $0,177^{*}$ & $-0,020$ & 0,025 & 0,004 & $0,233^{*}$ \\
\hline Brasil & $0,022^{*}$ & $0,161^{*}$ & $-0,009$ & $-0,183$ & 0,272 & $0,294^{+}$ \\
\hline \multicolumn{7}{|c|}{ Painel B: Período pós-Mercosul (janeiro de 1991 a dezembro de 1995) } \\
\hline Argentina & $-0,003$ & $0,127^{*}$ & $0,070^{+}$ & $0,621^{*}$ & $0,566^{\star}$ & $0,773^{\star}$ \\
\hline Brasil & $0,017^{*}$ & $-0,038$ & $0,233^{\star}$ & $0,619^{*}$ & $0,888^{\star}$ & $0,573^{\star}$ \\
\hline \multicolumn{7}{|c|}{ Painel C: Período total (janeiro de 1986 a dezembro de 1995) } \\
\hline Argentina & $0,012^{*}$ & $0,127^{\star}$ & 0,030 & $0,318^{*}$ & 0,153 & $0,324^{\star}$ \\
\hline Brasil & $0,021^{*}$ & $0,080^{+}$ & 0,191 & $0,267^{+}$ & $0,405^{+}$ & $0,380^{*}$ \\
\hline
\end{tabular}

Nota: Os fatores locais referem-se à primeira defasagem e à variação cambial. Os fatores globais dizem respeito aos mercados americano, chileno e ao mercado parceiro no Mercosul (Argentina, no caso do Brasil, e Brasil, no caso da Argentina). Todos os retornos estão na forma logarítmica. 
Figura 1 - Correlação anual do mercado brasileiro frente aos demais em moeda local para o período 1982-1995

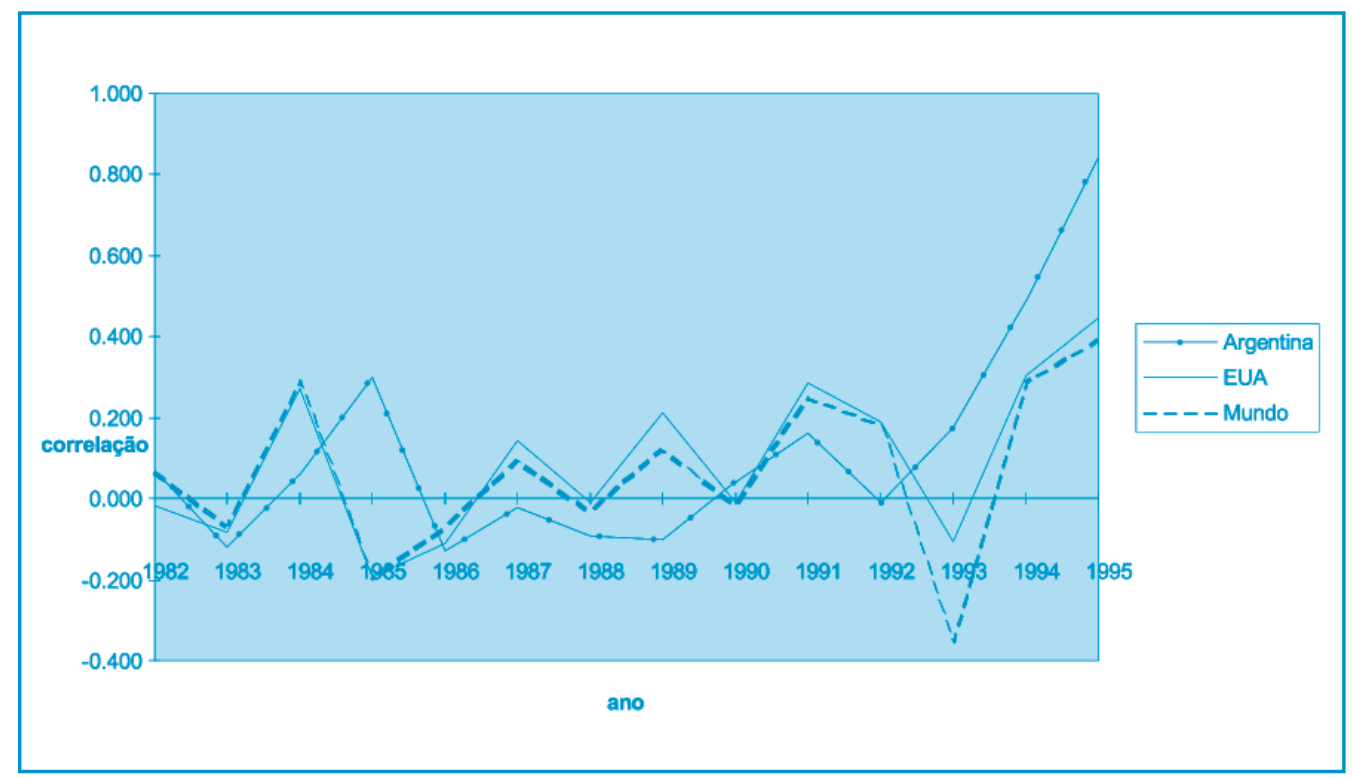

\section{CONCLUSÕES}

Nossos resultados apontam para uma maior integração entre os mercados acionários argentino e brasileiro mesmo depois do controle para possíveis elos com o mercado americano e chileno e para fatores locais como a variação cambial e a primeira autodefasagem. No período antecedente ao Mercosul (1986-1990), nenhum fator internacional, seja o mercado americano, seja o mercado chileno, ou o futuro mercado parceiro no Mercosul, apresentou uma relação significativa com o mercado argentino ou brasileiro. Em outras palavras, os mercados argentino e brasileiro encontravam-se segmentados e eram influenciados apenas por fatores locais.

A situação muda completamente no período posterior à assinatura do Tratado de Assunção. Os coeficientes dos três fatores internacionais tornam-se significativos tanto para a Argentina quanto para o Brasil. Nossa interpretação para esses resultados é que houve uma integração dos mercados argentino e brasileiro não somente através do mercado americano, como também através de fatores comuns a outros mercados da região e fatores comuns ao país parceiro no Mercosul, no caso, possivelmente a integração econômica resultante do acordo. A integração com o mercado americano e com o mercado chileno provavelmente se deve a fatores como a redução das restrições ao investimento estrangeiro, que, no caso da América Latina, vem primordialmente dos Estados Unidos, à emissão e negociação de títulos argentinos e brasileiros na forma de ADRs no mercado americano e ao crescente número de fundos de ações focalizando a região ou países da região. Os resultados que encontramos para os modelos de integração confirmam o que as correlações bivariadas crescentes já indicavam. No caso do cone sul, os elos entre os mercados se traduziram em uma maior integração regional.

A maior integração entre os mercados implica benefícios menores para o investidor internacional, em particular o americano, quando da utilização desses ativos na composição da carteira global. Em outras palavras: um problema na economia argentina pode acarretar queda, não só das ações argentinas como também das brasileiras e vice-versa. Esse tipo de fenômeno pôde ser observado após a crise cambial mexicana no final de 1994. Evidência casual recente indica que notícias referentes à permanência ou não do Ministro da Economia argentino ou problemas na balança comercial brasileira, por exemplo, são suficientes para causar grandes oscilações em ambas as bolsas. 